\title{
Virtual Brain: Model-Based Framework for Dependable EEG Sensing and Actuation in Intelligent Brain IOT System
}

\author{
P. Geetha ${ }^{1}$, R. Reni Hena Helan ${ }^{2}$, M. Nithya Sree ${ }^{3}$, B. Shifrah ${ }^{4}$, V. S. Yageetha ${ }^{5}$ \\ ${ }^{1,2}$ Assistant Professor, Dhanalakshmi College of Engineering, Chennai \\ ${ }^{3,4,5}$ Student, Dhanalakshmi College of Engineering, Chennai
}

\begin{abstract}
Real-time Brain IoT Systems are costly. But, developing a cheaper system has been accelerated greatly by the better research that is being done on Virtual Brain. The death of an important person on a secret mission is consider sensitive information and must be handled with as much security as possible. By ensuring this discreteness, the time taken for the message of their death to reach the relevant authority is increased to up to a few days. The time taken to deliver the message is too much. These days, the improvements in hardware increasing the abilities of the Virtual brain and of the wearable Brain IoT sensors have made the development of numerous new software frameworks possible for the developers to create useful applications that combine the human brain with IoT. Multiple sensory pathways are also enabled for communications of the human brain with larger sized data. The main aim of this project is to upload confidential documents onto the clouds securely as possible. Knowledge on how every individual think and recalls memory is found by the Virtual cerebrum undertaking. Both of the mentioned fields have been accelerated greatly in terms of development by these researches. This will in turn drive the need of an energy-efficient technology that supports the wider demands of the IoT. The brain activity extraction in IoT has been surveyed thoroughly in this project. This includes IoT models based on Electroencephalogram (EEG), machine learning and the current active platforms. The main findings of our survey highlights three major trends of development in the virtual brain development system, viz, IoT, EEG and cloud computing. Since the human body has the low frequency range of $7 \mathrm{~Hz}$ or below $7 \mathrm{~Hz}$, it cannot be detected by the EEG sensor. So, the wearable EEG sensor has a band that goes around the head. Once the individual is dead, the confidential information that is securely stored in the cloud can be sent to the mail id that was pre-registered by the user without any delay. This system is very time efficient.
\end{abstract}

Keywords: EEG sensor, AVR Microcontroller, IoT Modem, UART (universal asynchronous receiver transmitter), BCI (Brain Computer-Interface)

\section{INTRODUCTION}

Over the years, all expert and mass-showcase utilization situations including keen structure expanding, showcase the growing number of Internet of Thing (IoT) items and its diverse administrations. A good insight into how the brain of a human being works is derived from a complete picture of the structure of the brain. This knowledge can be used to discover new drugs and methods of treatment for a large array of brain disorders. The collection of a large volume of images of the brain tissues in the cellular and the subcellular level, has been made possible by the advancements in the intact brain imaging techniques. These include 
Magnified Analysis of the Proteome (MAP) and CLARITY. Though this seems beneficial, these high-resolution images pose another challenge; to efficiently process and analyses them. The figures released last on incomes and of sent de-indecencies, shows an estimation of the IoT arrangements in the actual scale operational circumstances. Moreover, pilot and business deployments are suggesting the importance of using the IoT principles in sophisticated utilization situations. The concept of Brain IoT revolves around the complex circumstances wherein the activation and control of a system are strengthened by the populaces of the IoT receptacles. The components that are used in this project are an EEG sensor, the AVR Microcontroller, the IoT Modem and the UART. 1. EEG (Electroencephalogram) is a sensor that is used to sense the electric pulses of the human brain., irrespective of the fact that the brain is active or not. 2. An AVR Microcontroller is used for using the fast indication handling activity that is present in an implanted framework., it already has all the features and is built onto a single chip. 3. BCI (Brain computer Interface) is the rapport of the Human Brain and the computer. 4. UART (Universal Asynchronous Receiver Transmitter) is used for transmitting and collecting the data and it's being pertained with the IoT. A handful are planned for a high throughput and a long neuron investigation at the phone degree, which is the basis for the understanding of the cerebral circuitry and for differentiating the substantial and toxic psyches. A high throughput and a low inactivity inquiry of mind information will require a fast database and programming interface that is acceptable to the tremendous scope of the diagrammatic inspection. Brain graphs offer a varied framework for the representation of the structural or serviceable topology in multiple categories. A multitude of software tools exist for analyzing the topology of brain systems utilizing a graph theory. They principally focus on researching the correlations of the anatomically insulated brain regions. Some are constructed for a high throughput and long-range neural analysis at the cellular level, which is crucial for comprehending the brain passages and for relating active and diseased brains. High throughput and low latency calculation of brain data will mandate high velocity databases and programming interfaces that are easily compatible to a big scale graph examination.

\section{LITERATURE REVIEW}

[1] N. M. Neihart and R. R. Harrison has spoken about the neural chronicle frameworks that need gadgets considering bidirectional information move. These gadgets should be little and low power as they are placed near to the cerebrum. Utilizing a parasitic radio wire under $2 \mathrm{~mm}$ long, a power level was estimated to be $59.73 \mathrm{~dB}$ m away off of one meter.

[2] R. Polana R. Nelson explains about BCI frameworks measure explicit highlights of mind action and make an interpretation of them into control flags that drive a yield. The sensors most normally been used in BCI contemplate have been electroencephalographic (EEG) chronicles from the scalp and single-neuron accounts from inside the cortex.

[3] B. Gosselin has introduced a method called BTC communication. BTC frameworks provides correspondence channel option for carrying out tasks and deducing in intelligent articles by means of orders sent by clients from their controlled mind exercise.

[4] L. H. Miranda and T. H. Meng describes about configurable, 3.6 to $7.5 \mathrm{GHz}$ beat UWB transmitter IC focused for neurological inserts with high information rate prerequisites. Each pattern of the RF beat is carefully programmable in plentifulness and span, empowering a truly adaptable forming of the transmitted PSD signal, without the utilization of a yield channel. EEG has been used in clinical and experimental settings for years. 
[5] Y. Gaoet al. describes about the Gait period analysis serves to determine the frequency and phase of each observed sequence so as to align sequences before matching. In this paper introduce a method called CMOS. A coordinated CMOS Ultra-Wideband remote telemetry handset for wearable and implantable clinical sensor applications is accounted for right now. This high obligation cycled, no coherent handset underpins adaptable information rate up to 10 $\mathrm{Mb} / \mathrm{s}$ with vitality effectiveness of $0.35 \mathrm{no} / \mathrm{bit}$ and $6.2 \mathrm{no} / \mathrm{bit}$ for transmitter and recipient, separately. Two main challenges are arising in the existing Internet of Things scenarios. One is the need to interconnect various unique platforms and smart Things in a single environment.

[6] Consumer-Grade EEG Measuring Sensors as Research Tools: A Review. Since the launch of the first consumer-grade EEG measuring sensors, NeuroSky Mindset, in 2007, competing manufacturers like NeuroSky, Emotiv, interaXon and OpenBCI have introduced at least one new device per year. All these products are affordable, portable, and easy to use despite the variations in make and versions. These features are unquestionably well-placed, given that one of the primary goals for their creation is to attract a new community of commercial users. Since conventional EEG has been used in clinical and experimental settings for years, the transition toward commercial and engineering sides has not been without uncertainty. With this in mind, researchers in related areas have been working diligently to ensure that these ostensibly innovative features were not implemented at the cost of reliability and accuracy, by performing validation studies comparing the output of data derived from consumer-grade EEG devices to information generated from standard research-grade counterparts.

[7] Ear-EEG-Based Objective Hearing Threshold Estimation Evaluated on Normal Hearing Subjects. The objective electroencephalogram (EEG) based technique of auditory steady-state response has been successfully used in the clinic to estimate hearing threshold levels (ASSR).

\section{Proposed Methodology}

Methodology basically is a model to clarify the strategies or methods used to configure, create or plan a project. This section clarifies about the product and equipment that will be utilized for building up this undertaking further. The outcomes need to be analyzed to accomplish the goal of this project.

\subsection{Overview of the system}

BCI (Brain-PC interface) approach will be executed in this paper. The outline of the project is exhibited below in Figure 1. The segments utilized in this task are EEG, AVR Micro regulator, IoT Modem, UART wire, and force supply is empowered. High speed signal processing is the primary use of this AVR miniature regulator. The AVR Micro regulator is associated with the EEG sensor and IoT modem. The transformer is utilized in this analysis since the 240v can be changed over into 5v. The transformer is associated with AVR Micro regulator. TFT (Thin Film Transmitter) is displayed above the micro regulator. It is utilized for the purpose to display. We should examine about the experimentation of the task. At the point when the force supply gets ON, it is needed to hold the EEG sensor on the forehead and contact is needed with hands. At the point when it is held, it results as Brain dynamic, if not then the mind isn't dynamic. So, in that TFT show, it shows as Brain dead. The virtual mind is quickening the improvement of reasonable ongoing cerebrum PC interface (BCI). Equipment prerequisites that increment the ability of the virtual mind are listed below:

The benefits of the proposed framework are: 
A reasonably priced innovation with good quality benefits.

$\checkmark$ Client family members get reminders on specific time.

$\checkmark$ Capacity to share current and saved data of the client.

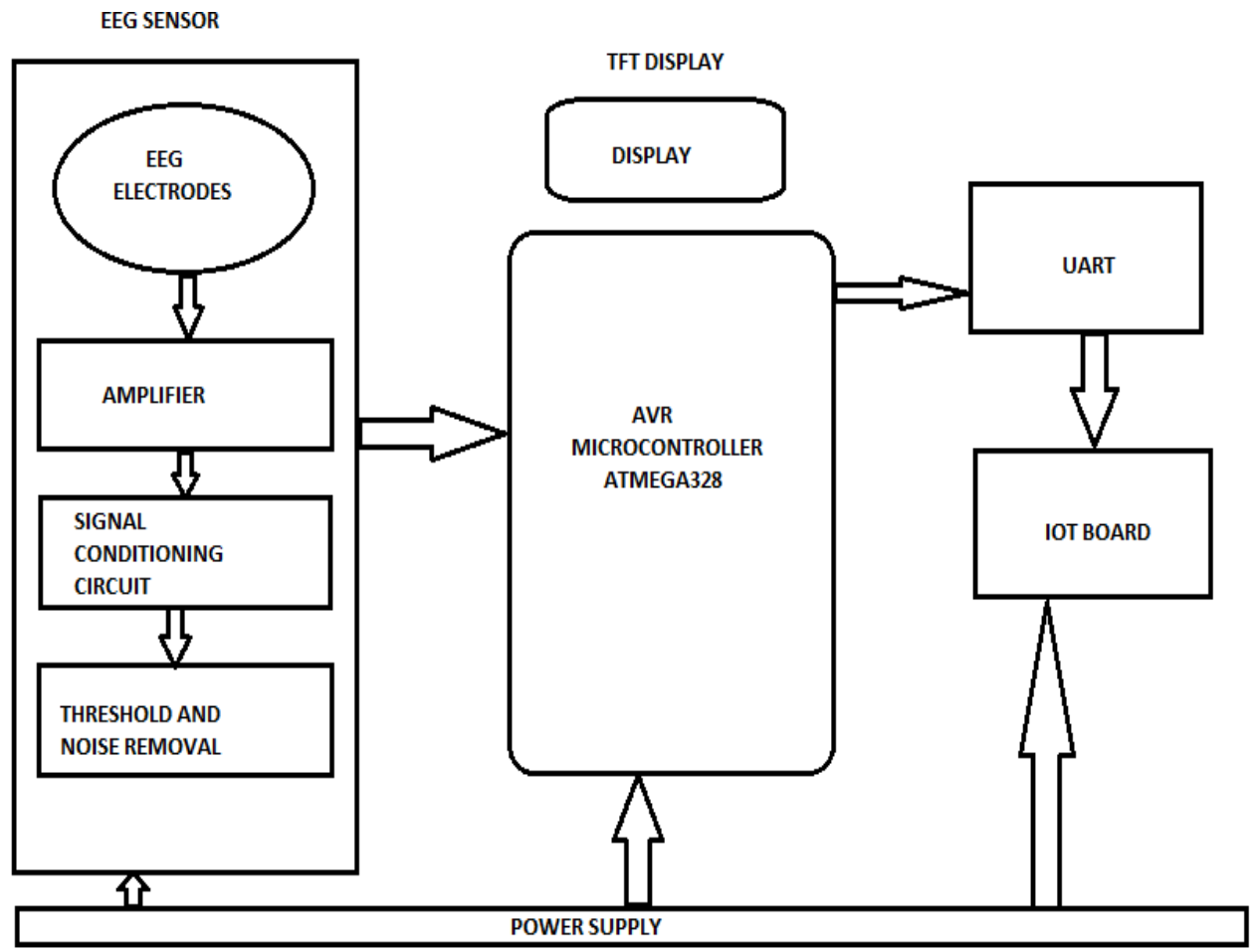

Figure 1. System Architecture

Coming up next are the modules that are separated into four dependents on the work done in the proposed framework shown in figure 3. The modules are,

1. Virtual cerebrum module

2. Privacy data module

3. Privilege to access module

4. Remainder module

\subsection{Virtual cerebrum module}

In this module, the components are getting coordinated. It comprises of parts like EEG (Electro Encephalogram), AVR microcontroller, IoT modem, Transformer, UART (Universal Asynchronous collector Transmitter) board, lastly a TFT show. The AVR microcontroller is associated with TFT show and transformer. UART board is associated with IoT modem and all these associated with power supply.

\subsection{Privacy data Module}

In this module, the main key is to enter the username and password in order to uncover this screen. By tapping the choices like document, mail id and telephone number, the client can register the things and save them away in the cloud. The data which stores in this module will be free from any privacy issues. 


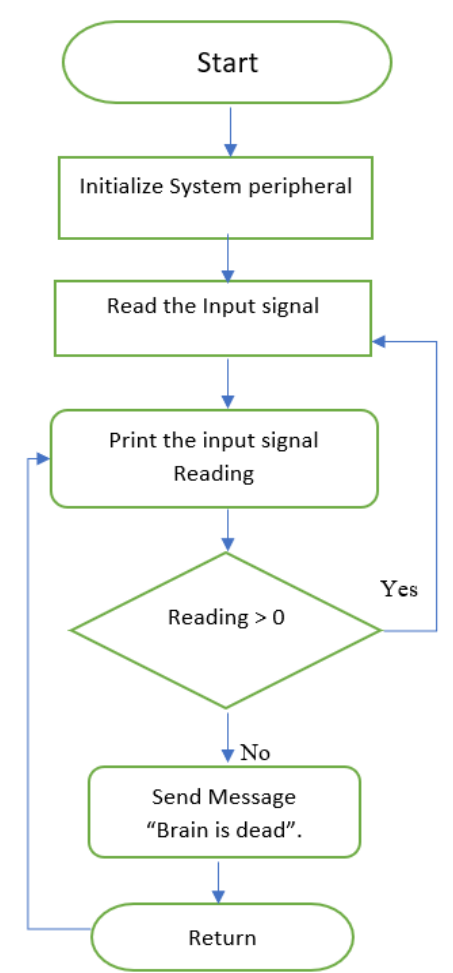

Figure 2. Flow Diagram

\subsection{Privilege to access module}

In this module, a customized username and password could be entered by the client. Basically, this is the module which is associated with the EEG (Electro Encephalogram) gadget and PC. By entering the username and the password, the client can go into the following screen. All clients should have an extraordinary username and password to protect their privacy. The client can enter data which needs to pass on to their family, companions or neighbor and can type in their customized message, alternatively, there is an option for them to save their data.

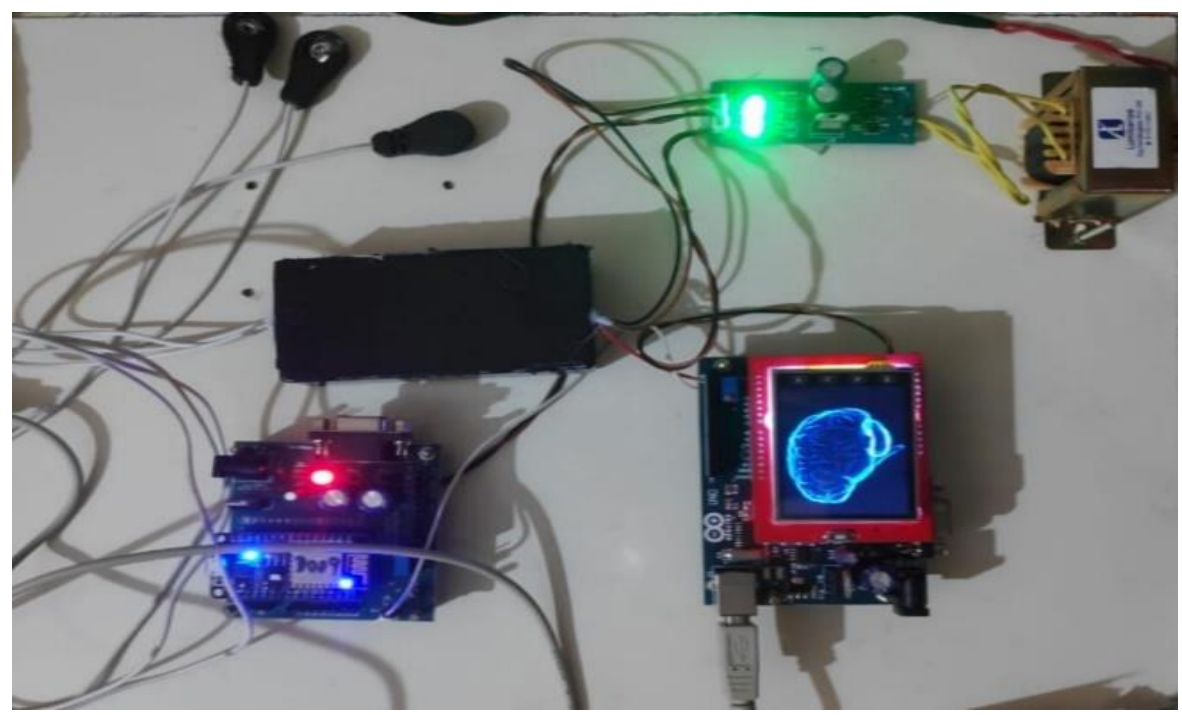

Figure 3. An image showing the proposed system 


\subsection{Remainder module}

At the point when the client inserts the privacy information on the cloud, the registered user will be notified via alert. The cloud is made such that it is highly secured and safe. On the off chance that a user or a client is not alive, all the saved data and information could be automatically forwarded to the enlisted mail id or mobile number.

\section{Components Details}

\subsection{EEG Sensor}

The basic work of the EEG sensor shown in Figure 4 is to quantify the electrical exercises which is created by the synchronized action of various neurons in volts which brings about an exceptional goal in terms of time, which makes us to discover the action inside the constraint of various divisions of cortical regions even at sub-second timescales. EEG, the quickest imaging procedures is accessible, and has high rate of sample. In the beginning, when EEG sensor was founded a numerous years ago, it was plotted on paper. These days in current frameworks, it carefully shows the information as a nonstop progression of voltages.

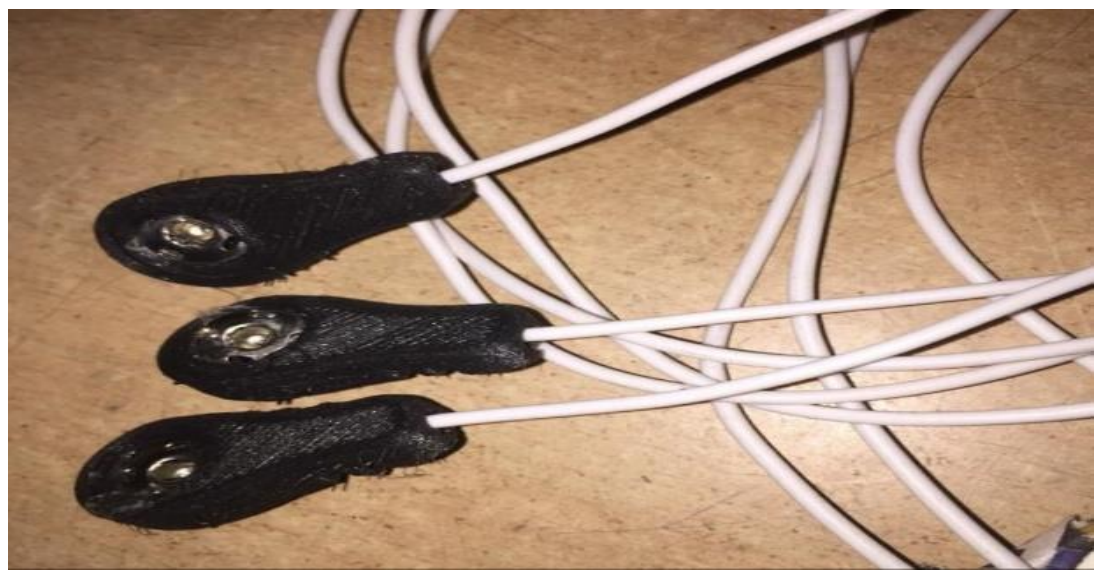

Figure 4. EEG Sensor

\subsection{EEG Signal}

EEG signal shown in Figure 5 is determined in terms of frequency and intensity. Signal intensity is estimated in microvolts $(\mu \mathrm{V})$. Signal recurrence of the human. EEG waves are of four kinds which are listed below:
$\checkmark$ theta
$\checkmark$ delta
$\checkmark$ alpha
$\checkmark$ beta

\subsection{Microcontroller - ARDUINO}

Arduino Microcontroller is one of the incredible single board PCs that have acquired exceptional Arduino is open-source, which means that software of programming and 
development is free. Spark fun is a decent source in Arduino equipment where US market is concerned. Arduino board can be utilized to make interface circuits to understand switches and different sensors, and to control tasks like engines and lights with less exertion.

\subsection{Arduino Uno Ide}

This is a cross-stage application (for Windows, macOS, Linux) that is written in the programming language Java and is utilized to compose and transfer projects to Arduino viable sheets, and other merchant improvement sheets with the assistance of third-party vendors. The source code for the IDE is delivered under the GNU General Public License, variant. The Arduino IDE upholds the languages $\mathrm{C}$ and $\mathrm{C}++$ utilizing some unique conditions with rules of code organizing.

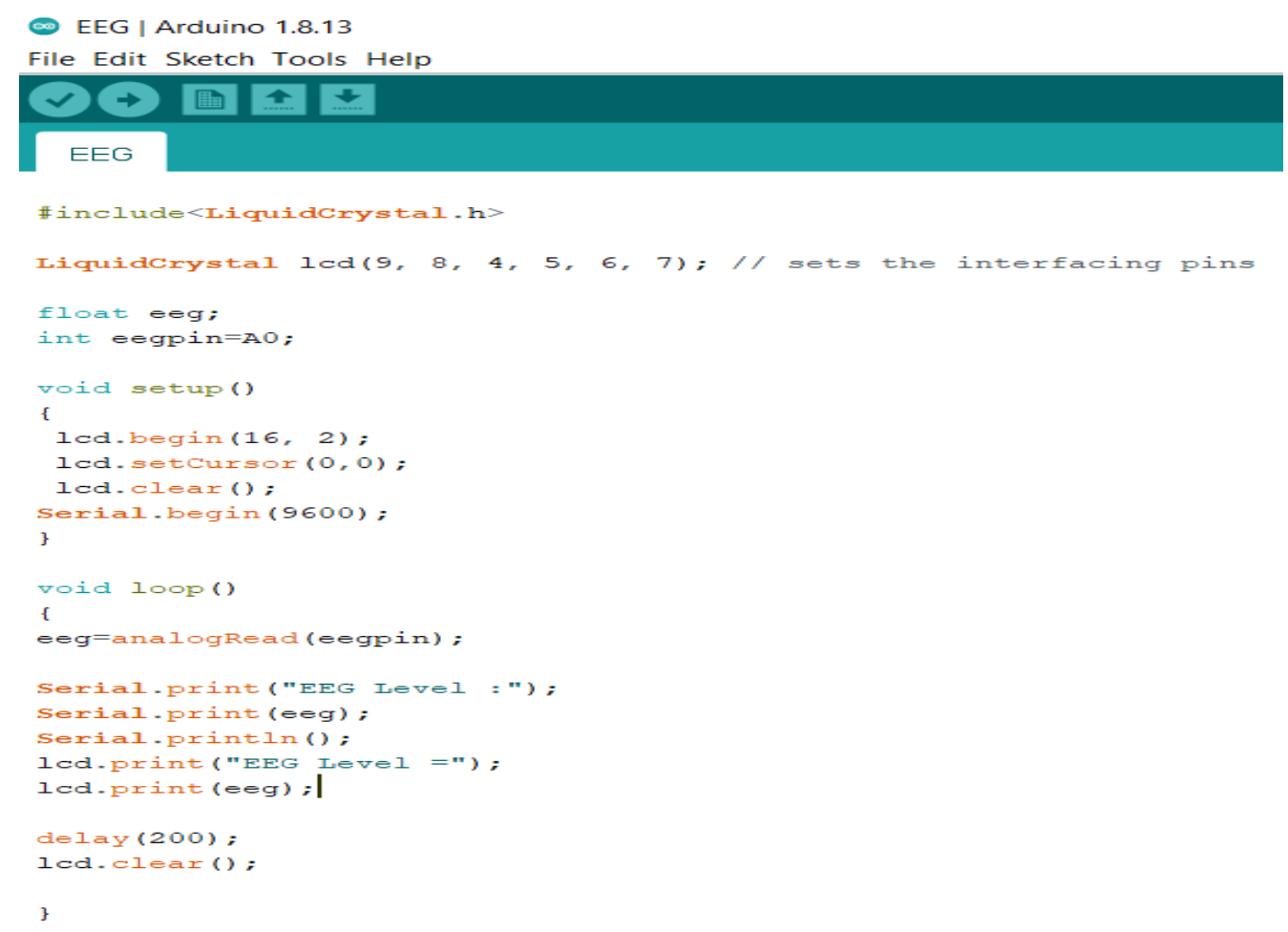

Figure 5. Arduino Code

\subsection{TFT Display}

TFT Touch Screen shown in Figure 7 is an Arduino with viable kaleidoscopic TFT show with viable impression. The TFT driver has proficient Driver IC with 8bit information and 4 bit control interface. We can draw message or do anything with the TFT library. It likewise has on board a miniature SD card opening on the rear of the screen and store bitmap pictures to show on the screen. The TFT library interfaces/connects with the regulator of the screen through SPI when utilizing the TFT library. Whether or not it is new development or old, every advancement radiates a particular level of ruinous X-bar release. All that presentations can cause eye strain if used for a long time with high brightness. Various presentations such as TFT lcd, drove, Amoled don't use electron shaft thought. The Arduino IDE upholds the languages C and $\mathrm{C}++$ utilizing some unique conditions with rules of code organizing. 


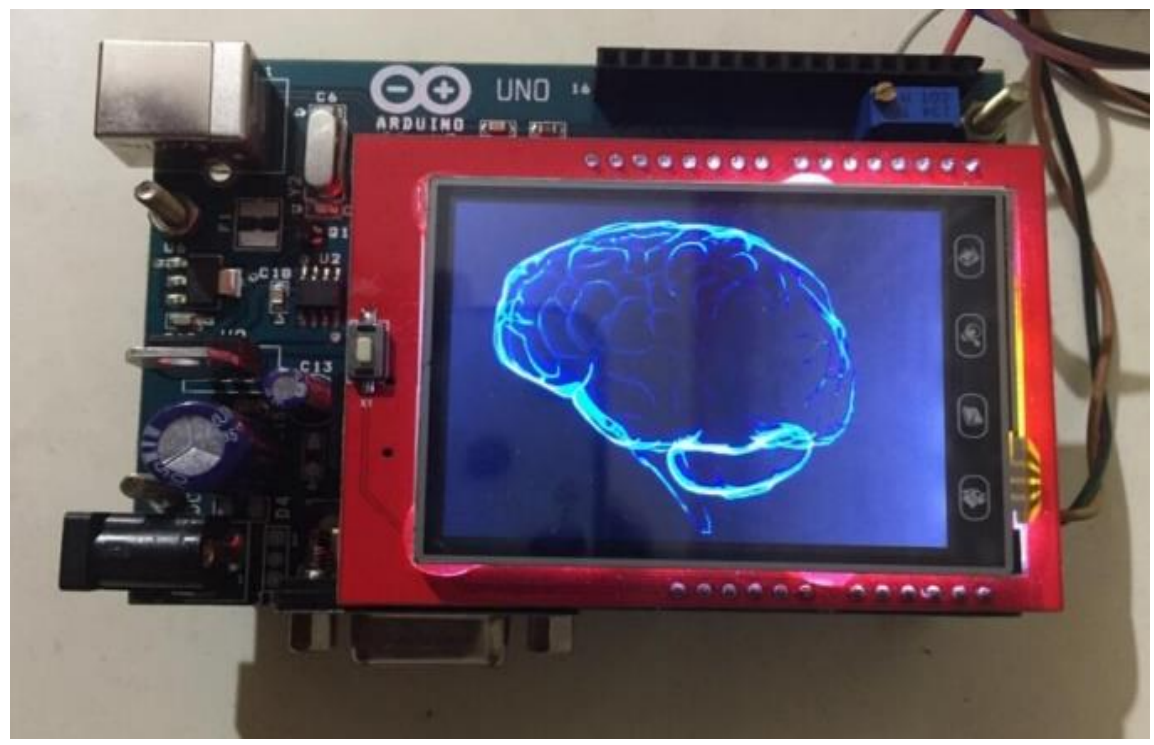

Figure 6. AVR Microcontroller Atmega328, Arduino Uno, TFT display

\subsection{ESP8266}

Wi-Fi Module, independent SOC (System on Chip)/incorporated circuits, chip with association with the TCP/IP convention stack that can give any unique microcontroller admittance to other Wi-Fi organization. The microcontroller which we are utilizing here in this undertaking is ARDUINO. The ESP8266 suits whether for facilitating an application or offloading all Wi-Fi organizing capacities from processor of different applications. This module accompanies AT orders firmware permits Arduino Wi-Fi shield. Community support in economy is growing rapidly. This module has on-board $80 \mathrm{MHz}$ low force $32 \mathrm{bit}$ processor and supports Bluetooth conjunction interfaces; it contains exceptional component of selfstandardized RF which works under every single working condition. And furthermore, it has APSD - (Automatic Power Save Delivery) with power saving component.

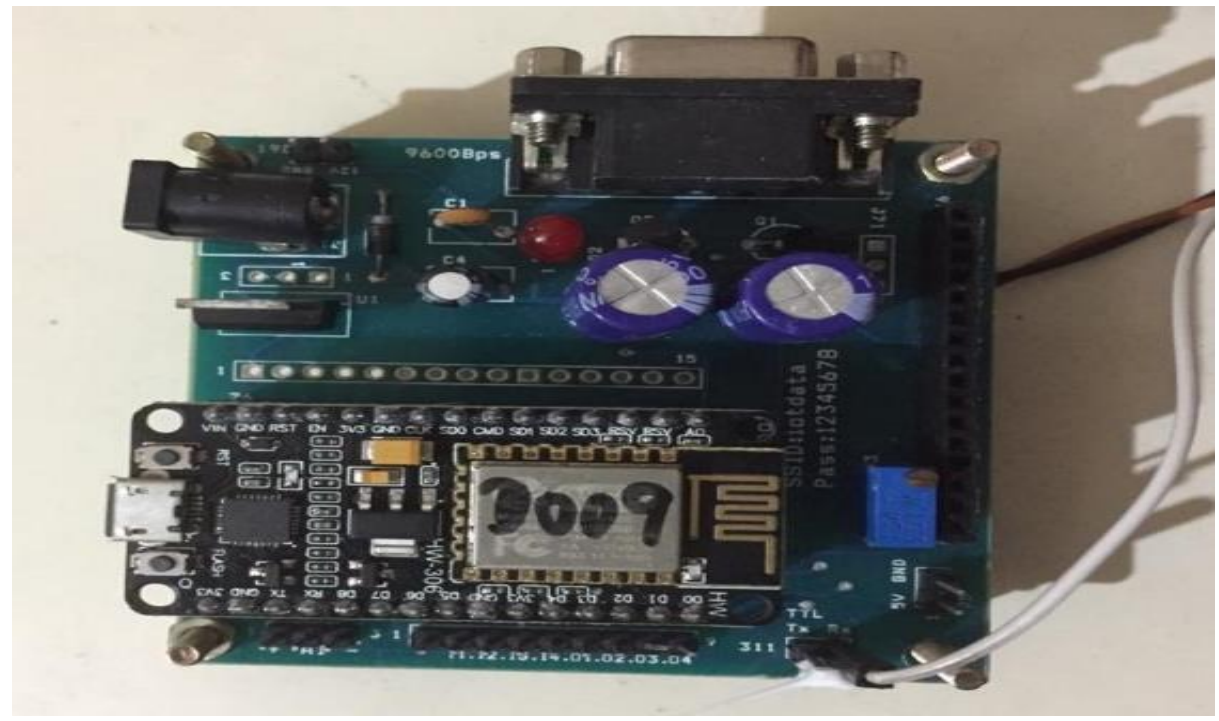

Figure 7. IOT Modem 
The software requirements used here is:

- PHP code.

\section{Implementation}

Here, initially the EEG headset with sensor is associated with the chip ARDUINO board and it is associated with the ESP8266 which is interconnected to the TFT Touchscreen. First the EEG headset sensors the EEG signal from the cerebrum with the terminals present in the headset and it checks whether it is alive or dead. Subsequent to detecting, given that the outcome comes is normal, it will be shown on the touch screen. In the event that we need in Software application, we can check the situation with the mind in ARDUINO IDE by utilizing the link associated with equipment unit and the gadget is to be introduced into the framework/PC in which Arduino programming has been introduced. In Tools menu, present in the Arduino application, click on the port which is empowered and after that we can straightforwardly see the situation with the cerebrum in the screen. In the event that any change or update is seen, the status will be sent through SMS and Email id for explicit individual in contact.

\subsection{Cloud computing}

Cloud computing infers taking care of and getting data and tasks over the Internet instead of your PC's hard drive. It is the mix of both equipment and programming. As this is clarified in this project, additionally we can join the gadget and web. Let's move on to the detailed explanation with regards to the software. Cloud computing, the act of utilizing a network of remote workers facilitated on the web to store, oversee, and process the information to local workers or PCs.

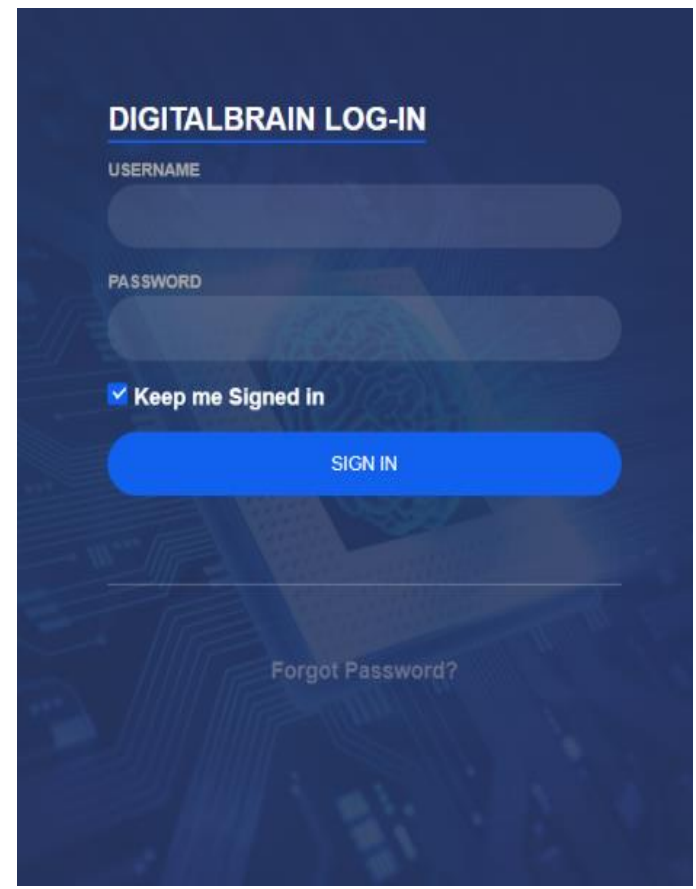

Figure 7. Login Page 
The snapshot shown in Figure 7 represents the login of the Digital brain. It comprises of a client name and login. The product side of the proposed framework is planned by utilizing a hypertext preprocessing code. It is a worker side scripting language, likewise it is simplicity of adjusting and doesn't include a lot of complexity. It is a backend language and in the proposed framework, it needs a lot of capacity, so hypertext preprocessing is able for that. As planned for a one-on-one page, it very well may be made, by joining all the pages through the hypertext preprocessing language. It can likewise be utilized as cost or liberated from cost depending upon the client.

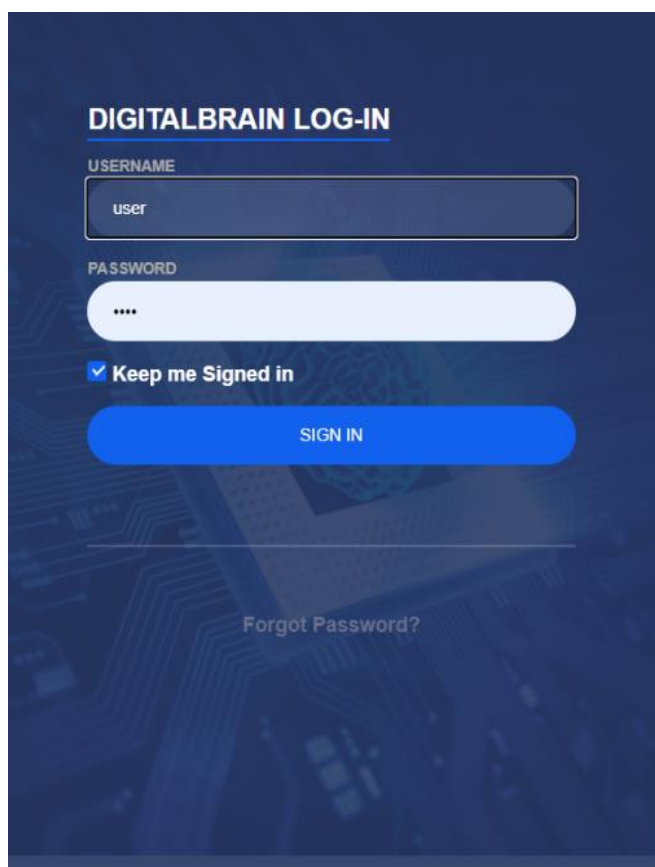

Figure 8. Sign-in Page

According to the standards, we can enter the username and the password. With the goal that it joins in and get into the following page. By utilizing PHP code, these sites and pages can be planned. Since it is a cloud computing it comprises of username and password and it can't be open by Third-party. We can safely make up the site and site while coding.

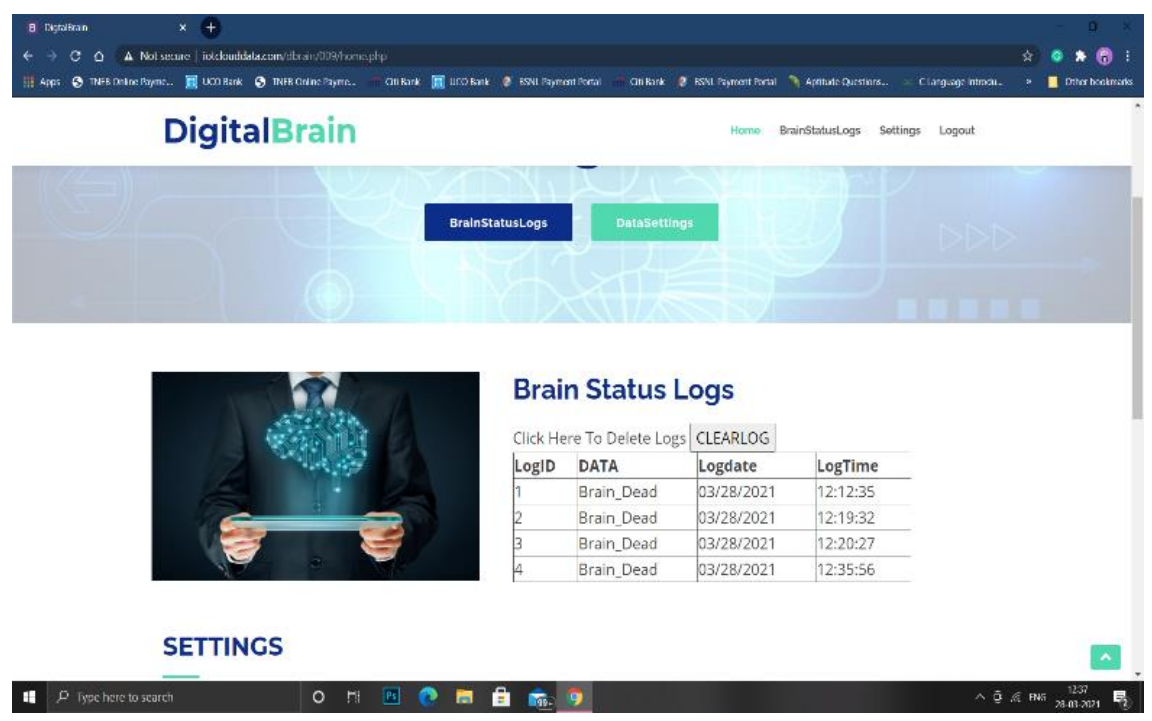

Figure 9. Brain Status Table with date and time 
The above snap in Figure 9 addresses the cerebrum status log subtleties of the client. At the point when the client is normal or when the client is dead, the information base precisely notices the date and time of the clients. For instance, on the date of March 28, the client must have worn the gadget and show the normal. Also, the other model the client's cerebrum is dead or the individual is no more, the status log is get refreshed with the precise date and time. The status data set gets refreshed as needs be.

\section{DigitalBrain Home Brainstatuslogs Settings Logout}

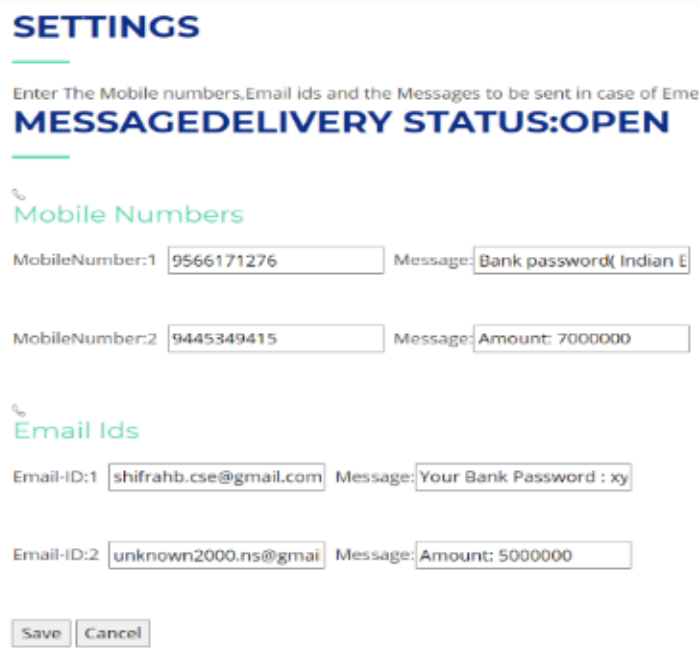

Figure 10. Upload of documents through phone number and mail id

\section{Result and Discussion}

In this proposed framework, the outcome can precisely ship off to the client's mail id or telephone number. But in the scenario that the client is alive, the client uses to type the data which he/she need to pass on to the top choice or specific individual. As the outcome plainly shows it is the BCI (BrainComputer Interface). Wearable gadgets have made conceivable a few new programming structures for engineers to utilize and make applications joining BCI and IoT. The archive or data can be effectively obtained by a specific individual immediately. In that note, this proposed framework is more effective.

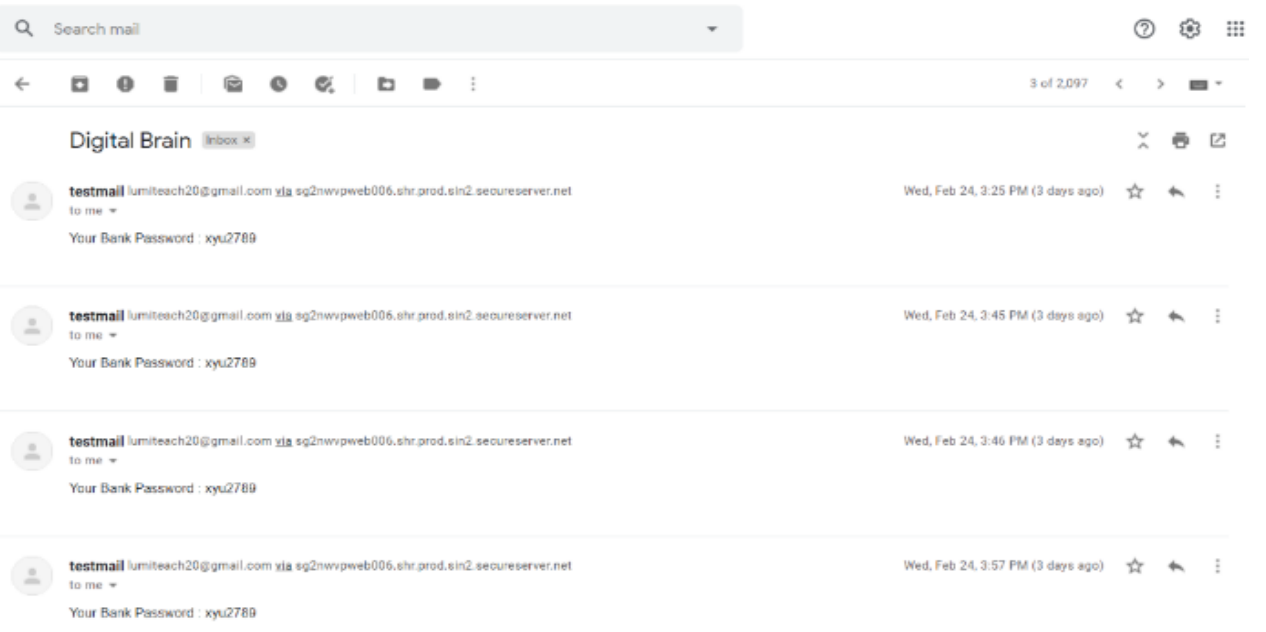

Figure 11. Message received by the user 
The snapshot shown in Figure 11 unmistakably clarifies that once the cerebrum isn't dynamic, it consequently ships off to the enrolled client.

\section{Conclusion \& Future Enhancement}

In this paper, we depicted a cloud-based framework for performing huge scope analysis of brain connectivity. We exhibited that our methodology can accomplish quick information collection and extraction for examination and analysis. There are numerous roads for future work in this domain. We clarify the digital brain and PC interface with great depth. Likewise, we present a technique called BCI (Brain-PC interface) model. This project or task builds up and establishes that human recollections and archives put away in the cloud are naturally shipped off to the enrolled client even the approved individual is no more. To start with, we might want to upgrade the web GUI by making it more intelligent and easier to use. After the demise of the human cerebrum, this cloud act has the human. This venture is basically intended for a wide range of individuals. Further, we plan to scale up to handle a lot bigger datasets (terabytes or more) with the objective of one day having the option to perform such investigation on the human cerebrum. We are additionally investigating the utilization of a poly store data set like Big DAWG as an information. Here we are utilizing logid for each client, through that they tend to get their information. In future, thumb impression for each client can be added rather than logid. With this logid, abuse of information is conceivable. Additionally, this extraordinary pack can be changed over into a chip and can embed either inside or remotely. For mentally challenged individuals and ill individuals this can be embedded inside. And furthermore, every individual can utilize this in everyday life and can escape from unplanned health problems and furthermore from phonies being managed without the specific individual's information. Amnesia patients will be utilized in particular. Later on, it will be created without power supply and furthermore rather than login they can make use of finger prints. When contrasted with username and passwords, the unique finger impression will be valuable and vital for individuals.

\section{REFERENCES}

[1] N. M. Neihart and R. R. Harrison "Micro power circuits for bidirectional wireless telemetry in neural recording applications, " IEEE Internet Computing, vol. 19, no. 4, (2018), pp. 60-67

[2] R. Polana R. Nelson, “Detecting Activities interface in Neural engineering”, Springer, (2018), pp. 85-121

[3] B. Gosselin, "Using brain-computer interface and internet of things to improve healthcare for wheelchair users.", (2017), pp. 80-181

[4] L. H. Miranda and T. H. Meng, "A programmable pulse UWB transmitter with $34 \%$ energy efficiency for multichannel neuro recording systems," Applications and Services (Healthcom), 2017 IEEE 19th International Conference on. IEEE, (2017), pp. 1-5

[5] Y. Gaoet al, "Low-power ultrawideband wireless telemetry transceiver for medical sensor applications" Applications and Services (Healthcom), 2017 IEEE 19th International Conference on. IEEE, (2017), pp. 1-5

[6] J. del R. Millàn and J. Mourino, "Asynchronous BCI and local neural classifiers: An overview of the Adaptive Brain Interface project,” IEEE Trans. Neural. Syst. Rehab. Eng., vol. 11, no. 2, pp. 159-161, Jun (2003)

[7] J. Borisoff, S. Mason, A. Bashashati, and G. Birch, "Brain-computer interface design for asynchronous control applications: Improvements to the LF-ASD asynchronous brain switch, " IEEE Trans. Biomed. Eng., vol. 51, no. 6, pp. 985-992, Jun (2004)

[8] R. Scherer, G. Müller, C. Neuper, B. Graimann, and G. Pfurtscheller, "An asynchronously controlled EEGbased virtualkeyboard: Improvement of the spelling rate,” IEEE Trans. Biomed. Eng., vol. 51, no. 6, pp. 979-984, Jun (2004) 
[9] G. Müller-Putz, R. Scherer, G. Pfurtscheller, and R. Rupp, "EEG-based neuroprosthesis control: A step towards clinical practice," Neurosci. Lett. vol. 382, no. 1-2, pp. 169-174, (2005). [Online]. Available: http://dx.doi.org/10.1016/j.neulet.2005.03.21.

[10] K.-R. Müller and B. Blankertz, "Toward noninvasive brain computer interfaces," Signal Process. Mag., vol. 23, no. 5, pp. 125-128, (2006)

[11] B. Blankertz, G. Dornhege, M. Krauledat, K.-R. Müller, V. Kunzmann, F. Losch, and G. Curio, "The Berlin Brain-Computer Interface: EEGbased communication without subject training," IEEE Trans. Neural. Syst. Rehab. Eng., vol. 14, no. 2, pp. 147-152, June (2006)

[12] G. Pfurtscheller, G. R. Müller-Putz, A. Schlögl, B. Graimann, R. Scherer, R. Leeb, C. Brunner, C. Keinrath, F. Lee, G. Townsend, C. Vidaurre, and C. Neuper, "15 years of BCI research at Graz University of Technology: Current projects,” IEEE Trans. Neural. Syst. Rehab. Eng., vol. 14, no. 2, pp. 205-210, June (2016)

[13] C. Vidaurre, A. Schlögl, R. Cabeza, R. Scherer, and G. Pfurtscheller, "Study of on-line adaptive discriminant analysis for eeg-based brain computer interfaces," IEEE Trans. Biomed. Eng., vol. 54, no. 3, pp. 550-556, March (2017)

[14] A. Schlögl, C. Keinrath, D. Zimmermann, R. Scherer, R. Leeb, and G. Pfurtscheller, "A fully automated correction method of EOG artifacts in EEG recordings, " Clin. Neurophysiol., vol. 118, pp. 98-104, (2017)

[15] Troyk P. R. and DeMichele G. A., "Inductively-coupled power and datalink for neural protheses using a classE oscillator and FSK modulation," in Proc. 2003 Int. Conf. IEEE Engineering in Medicine and Biology Society, Cancún, Mexico, Sep. (2003), pp. 3028-3031.

[16] Voigt P. and Von A. Bussche dem, "The EU General Data Protection Regulation (GDPR)," A Practical Guide, 1st Ed., Cham: Springer International Publishing, (2017) 